\title{
Article
}

Mycosphere

\section{Curvularia mosaddeghii sp. nov., a novel species from the family Pleosporaceae}

\section{Heidari $\mathbf{K}^{1}$, Mehrabi-Koushki $\mathbf{M}^{1,2 *}$ and Farokhinejad $\mathbf{R}^{1}$}

${ }^{1}$ Plant Protection Department, Agriculture Faculty, Shahid Chamran University of Ahvaz, Ahvaz, Iran

${ }^{2}$ Biotechnology and Bioscience Research Center, Shahid Chamran University of Ahvaz, Ahvaz, Iran

Heidari K, Mehrabi-Koushki M, Farokhinejad R 2018 - Curvularia mosaddeghii sp. nov., a novel species from the family Pleosporaceae. Mycosphere 9(4), 635-646, Doi 10.5943/mycosphere/9/4/2

\begin{abstract}
The new species $C$. mosaddeghii sp. nov. isolated from plants of Syzygium cumini and Vigna unguiculata is described and illustrated. Three-locus DNA sequence based phylogeny, in combination with morphology of the asexual morph, were used to characterize this species. Phylogenetic analysis used combined sequences of internal transcribed spacer regions $1 \& 2$ and 5.8S nrDNA (ITS), partial glyceraldehyde-3-phosphate dehydrogenase $(G P D H)$ and part of the translation elongation factor $1-\alpha(E F 1 \alpha)$. In the phylogenetic trees, both isolates of $C$. mosaddeghii clustered together as a monophyletic clade with strong support, distinct from other previously known species of Curvularia. Morphologically, this species is distinguished from closely related species by having narrower conidia and hila.
\end{abstract}

Key words - Ahvaz - Jambolan - Cowpea - Mycoflora - New species

\section{Introduction}

Curvularia belongs to Pleosporaceae and is widely distributed in soil water and plants and infects humans and animals (Sivanesan 1987, Manamgoda et al. 2011, 2012 a, b, da Cunha et al. 2013, Rangaswamy et al. 2013, Verma et al. 2013, Hyde et al. 2014, Ariyawansa et al. 2015, Wijayawardene et al. 2017, 2018). Curvularia species are mainly saprobes often found in an association with hosts and non-host living organisms in tropical regions (Manamgoda et al. 2011, 2012a, b, 2014, 2015, Scott \& Carter 2014, Tan et al. 2014), some are also opportunistic pathogens throughout the world (Manamgoda et al. 2011, Madrid et al. 2014). Some species are endophytes in various plant species (Tadych et al. 2012, Gautam et al. 2013, Jena \& Tayung 2013).

Curvularia was established by Boedijn in 1933, with the description of $C$. lunata (Wakker) Boedijn (Boedijn 1933) as the type species. Taxonomic classification of helminthosporium-like fungi based on morphological characteristics is insufficient and artificial, and species identification can only be elucidated using molecular studies (Manamgoda et al. 2012a, b, 2014, 2015, Madrid et al. 2014, Tan et al. 2014, Tomaso-Peterson et al. 2016, Marin-Felix et al. 2017a, b). In order to resolve phylogenetic relationships and improve the systematics of Curvularia and Bipolaris species, the nucleotide sequences of ITS (internal nuclear ribosomal transcribed spacer region), GPDH (glyceraldehyde-3-phosphate dehydrogenase) and EFla (translation elongation factor 1-a) were used exclusively or in combination for species delimitation (Manamgoda et al. 2012a, b, 2014, 2015, Madrid et al. 2014, Tan et al. 2014, Tomaso-Peterson et al. 2016, Marin-Felix et al. 2017a, b). According to the most recent phylogenetic analysis of Bipolaris- and Curvularia-like 
taxa (Manamgoda et al. 2015, Tomaso-Peterson et al. 2016, Marin-Felix et al. 2017a, b), Curvularia was emended as a monophyletic genus to accommodate 75 currently validated and five unknown species.

In the present study, a new species of Curvularia isolated from Iran is described through polyphasic approaches. A multi-locus phylogeny (ITS, GPDH and EFI $\alpha$ ), combined with the morphological analysis of the asexual morph, was used to separate these unknown species from previously described species.

\section{Materials \& Methods}

\section{Isolates and typification}

Two Curvularia isolates identified in this study were recovered from leaf spot of jambolan (Syzygium cumini) and healthy roots of cowpea (Vigna unguiculata) growing in Ahvaz in the southwest of Iran. The type specimens (dried cultures) are deposited in Herbarium Ministerii Iranici Agriculturae, Iranian Research Institute of Plant Protection, Tehran, Iran (IRAN 16938F). The extype living culture and other isolate under survey are deposited in the Iranian Fungal Culture Collection, Iranian Research Institute of Plant Protection, Tehran, Iran (IRAN 3131C and IRAN 3123C). In addition, they are kept in Collection of Fungal Cultures, Department of Plant Protection, Shahid Chamran University of Ahvaz, Iran (SCUA-Ahv-Syz-P and SCUA-Ahw-Vig-1Rb) (Table 1).

\section{Morphological and physiological study}

The morphological characteristics of asexual morph were examined using 3-day to 14-dayold cultures grown on potato dextrose agar (PDA, Merck) at $28{ }^{\circ} \mathrm{C}$ in a 12 -h fluorescent light-anddark. These characteristics (including those of the mycelia, conidiophore, and conidia) were studied using the method of Riddle and Briggs (1950). The colour was determined as described in Methuen handbook of color (Kornerup \& Wanscher 1967). The slide cultures were prepared according to the protocol described by Beneke \& Rogers (1996). Sixty measurements for each character were made with the 40x and 100x objective lens of a Leitz wetzlar (SM-LUX) Basic Biological Light Microscope. The photomicrographs were recorded with an OLYMPUS BX51 microscope fixed with an OLYMPUS DP12 digital camera. The isolates were preliminarily identified based on morphology documented in literature review (Manamgoda et al. 2012a, b, 2014, 2015, Madrid et al. 2014, Tan et al. 2014, Tomaso-Peterson et al. 2016, Marin-Felix et al. 2017a, b). The rate of mycelial growth was determined at $28{ }^{\circ} \mathrm{C}$.

\section{Fungal biomass preparation}

Single spore cultures of each Curvularia isolates were inoculated into Erlenmeyer flasks containing potato-dextrose-broth medium. The flasks were incubated at $180 \mathrm{rpm}$ for 10-15 days at $28{ }^{\circ} \mathrm{C}$ to achieve the maximum growth of fungi. The mycelial biomass was collected and washed by passing through filter papers using sterile distilled water. The mycelia were freeze-dried (FreezeDryer, Alpha 1-2LD Plus, Christ) and powdered in the mortar containing liquid nitrogen. The mycelia powders was collected into $10 \mathrm{ml}$ tubes and stored in the $-20^{\circ} \mathrm{C}$ freezer until consumed.

\section{DNA extraction and amplification}

Fungal DNA was isolated from $100 \mathrm{mg}$ of freeze-dried mycelia using a Phenol:chloroform based protocol as previously described by Raeder \& Broda (1985), with some modification (Ahmadpour et al. 2017). Mycelial lysates was extracted two times by Phenol:chloroform:isoamyl alcohol, followed by extracting with chloroform:isoamyl alcohol. DNA samples were analyzed using a spectrophotometer (eppendorf BioPhotometer plus) and loading on the gel. Partial regions of nrDNA (internal transcribed spacer regions $1 \& 2,5.8 \mathrm{~S}, \mathrm{LSU}-\mathrm{D} 1 / \mathrm{D} 2), G P D H$ and EF1 $\alpha$ were amplified with a thermal cycler (MJ Mini ${ }^{\text {TM }}$ Gradient Thermal Cycler) using primers ITS1 and NL4 (White et al. 1990), gpd1 and gpd2 (Berbee et al. 1999) and EF1983 and EF12218R, respectively 
Table 1 Strains used in this study and their GenBank accession numbers. The sequences of newly described species are indicated in bold.

\begin{tabular}{|c|c|c|c|c|c|c|}
\hline \multirow[t]{2}{*}{ Species name } & \multirow{2}{*}{$\begin{array}{l}\text { isolate name } \\
\text { strain no." }\end{array}$} & \multirow{2}{*}{ or Source or host } & \multirow[t]{2}{*}{ origin } & \multicolumn{3}{|c|}{ GenBank Accession numbers } \\
\hline & & & & ITS & $G P D H$ & $E F 1 \alpha$ \\
\hline B. drechsleri & MUS0028 & $\begin{array}{l}\text { Microstegium } \\
\text { vimineum }\end{array}$ & USA & KF500532 & KF500535 & KM093761 \\
\hline C. aeria & CBS 294.61 & Air & Brazil & HE861850 & HF565450 & - \\
\hline C. affinis & BS 154.34 & Unknown & Indonesia & KJ909780 & KM230401 & 1 KM19656 \\
\hline C. affinis & BS 185.49 & - & - & HG778982 & 2 HG779127 & $7-$ \\
\hline C. akaii & CBS 317.86 & Themada triandra & Japan & KJ909782 & KM230402 & 2 KM196569 \\
\hline C. akaiiensis & BRIP 16080 & - & - & KJ415539 & KJ415407 & KJ415453 \\
\hline C. alcornii & MFLUCC 100703 & Zea & Thailand & JX256420 & JX276433 & JX266589 \\
\hline C. americana & UTHSC 072649 & Toe tissue & USA & HE861834 & HF565486 & - \\
\hline C. americana & UTHSC 08 & Peritoneal dialysis & & HE861832 & HF565487 & - \\
\hline C. asianensis & MFLUCC 1 & Sacc & lailand & $\mathrm{JX} 256425$ & JX276437 & JX266594 \\
\hline C. asianensis & MFLUCC 100711 & Panicum sp. & Thail & JX256424 & JX276436 & JX266593 \\
\hline C. australiensis & BRIP 12044 & Oryza sativa & - & KJ415540 & KJ415406 & KJ415452 \\
\hline C. australiensis & CBS 172.57 & Ory & Vietnam & JN601026 & JN601036 & JN601003 \\
\hline C. australis & BRIP 12247a & $\begin{array}{l}\text { Eragrostis } \\
\text { cilianensis }\end{array}$ & Australia & KC424609 & KC747759 & KC503954 \\
\hline C. australis & BRIP 12521 & Sporobolus carolii & - & KJ415541 & KJ415405 & KJ415451 \\
\hline C. bannonii & BRIP 16732 & $\begin{array}{l}\text { Jacquemontia } \\
\text { tamnifolia }\end{array}$ & USA & KJ415542 & KJ415404 & KJ415450 \\
\hline C. bannonii & DAOM196762 & Tent wall & USA & KP400634 & KP419983 & KP735688 \\
\hline C. borreriae & AR5176r & Sorghum bicolor & $\begin{array}{l}\text { South } \\
\text { Africa }\end{array}$ & KР400637 & KP419986 & KP735690 \\
\hline C. borreriae & MFLUCC 11-0422 & & & KP400638 & KP419987 & KM196571 \\
\hline & & & & $\mathrm{KJ} 4$ & $\mathrm{KJ} 4$ & 5449 \\
\hline$r a$ & $\mathrm{CB}$ & So & & KJ922372 & $\mathrm{KM}$ & 230405 \\
\hline & & - & - & HM053667 & $7 \mathrm{HM}$ & \\
\hline & & Buchlo & & KJ9 & $\mathrm{KM}$ & 9 KM19658 \\
\hline payae & & & & $\mathrm{HG}^{\prime}$ & $4 \mathrm{HG}$ & $5-$ \\
\hline hlamydospora & UTH & & & $\mathrm{HG}^{\prime}$ & I HG & $1-$ \\
\hline avata & BRII & & & KU & & $\mathrm{KL}$ \\
\hline & CBS & & & JN1 & JN60 & JN601006 \\
\hline C. crustacea & BRIP 13524 & bolus sp. & Indonesia & KJ415544 & KJ415402 & KJ415448 \\
\hline $\begin{array}{l}\text { C. } \\
\text { dactyloctenicola }\end{array}$ & CPC 28810 & $\begin{array}{l}\text { Dactyloctenium } \\
\text { aegyptium }\end{array}$ & Thailand & MF490815 & MF490837 & MF490858 \\
\hline C. dactyloctenii & BRIP 12846 & $\begin{array}{l}\text { Dactyloctenium } \\
\text { radulans }\end{array}$ & Australia & KJ415545 & KJ415401 & KJ415447 \\
\hline C. ellisii & CBS 193.62 & $\mathrm{Ai}$ & & JN192375 & JN600963 & JN601007 \\
\hline lisii & IN & $\mathrm{Ai}$ & Pakistan & KJ922379 & KM061792 & $2-$ \\
\hline ragrostidis & CBS & - & - & HG778986 & 5 HG779154 & $1-$ \\
\hline eniculata & CBS & Unkn & Indonesia & KJ909781 & KM0 & 9 КМ2304 \\
\hline C. gladioli & CBS 210.79 & - & - & HG778987 & 7 HG779123 & - \\
\hline C. gladioli & ICMP 6160 & Gladiolus sp. & $\begin{array}{l}\text { New } \\
\text { Zealand }\end{array}$ & JX256426 & JX276438 & JX266595 \\
\hline C. graminicola & BRIP 23186a & - & & JN192376 & JN600964 & JN60 \\
\hline & & Triticum aestivl & & & & KJ415446 \\
\hline C. hawaiiensis & BRIP 11987 & Oryza sativa & USA & KJ415547 & KJ415399 & KJ415445 \\
\hline $\begin{array}{l}\text { C. } \\
\text { heteropogonicola }\end{array}$ & BRIP 14579 & $\begin{array}{l}\text { Heteropogon } \\
\text { contortus }\end{array}$ & India & KJ415548 & KJ415398 & KJ415444 \\
\hline C. heteropogonis & CBS 284.91 & $\begin{array}{l}\text { Heteropogon } \\
\text { contortus }\end{array}$ & Australia & JN192379 & JN600969 & JN601013 \\
\hline C. heteropogonis & CBS 511.91 & - & - & HF934918 & HG779122 & $2-$ \\
\hline
\end{tabular}


Table 1 Continued.

\begin{tabular}{|c|c|c|c|c|c|c|}
\hline \multirow[t]{2}{*}{ Species name } & \multirow{2}{*}{\multicolumn{2}{|c|}{$\begin{array}{l}\text { isolate name or strain Source or host } \\
\text { no." }\end{array}$}} & \multirow[t]{2}{*}{ origin } & \multicolumn{3}{|c|}{ GenBank Accession numbers } \\
\hline & & & & ITS & GPDH & $E F 1 \alpha$ \\
\hline C. hominis & AR 5118 & Lolium perene & USA & KP400639 & KP419988 & KM196580 \\
\hline C. hominis & MFLUCC 120191 & Unknown grass & Thailand & KP400640 & KP419989 & KM196581 \\
\hline C. homomorpha & CBS 156.60 & - & - & JN192380 & JN600970 & JN601014 \\
\hline C. inaequalis & CBS 102.42 & Sand dune soil & France & KJ922375 & KM061787 & 7 KM196574 \\
\hline C. inaequalis & DAOM 20022 & Pisum sativum & Canada & KJ922374 & KM061786 & 6 KM196575 \\
\hline C. intermedius & CBS 334.64 & - & - & HG778991 & HG779155 & - \\
\hline C. intermedius & UTHSC 09-3240 & - & - & HE861855 & HF565469 & - \\
\hline C. ischaemi & CBS 630.82 & - & - & JX256428 & JX276440 & - \\
\hline C. ischaemi & ICMP 6172 & $\begin{array}{l}\text { Ischaemum } \\
\text { indicum }\end{array}$ & $\begin{array}{l}\text { New } \\
\text { Zealand }\end{array}$ & JX256428 & JX276440 & - \\
\hline C. lunata & CBS 730.96 & Lung biopsy & USA & JX256429 & JX276441 & JX266596 \\
\hline C. lunata & CBS 157.34 & $\begin{array}{l}\text { Unknown } \\
\text { substrate }\end{array}$ & Indonesia & $\mathrm{JX} 256430$ & JX276442 & JX266597 \\
\hline C. malina & CBS 131274 & zoysiagrass & USA & JF812154 & KP153179 & KR493095 \\
\hline C. malina & FLS- & Bermuda grass & USA & KR493070 & KR493083 & KR493093 \\
\hline C. miyakei & CBS197.29 & Eragrostis pilosa & Japan & KJ909770 & KM083611 & 1 KM196568 \\
\hline C. mosaddeghii & $\begin{array}{l}\text { IRAN 3131C; SC } \\
\text { Ahv-Syz-P }\end{array}$ & $\begin{array}{l}- \text { Syzygium } \\
\text { cumini }\end{array}$ & Iran & MG84673 & MH392155 & 5 MH39215 \\
\hline C. mosaddeghii & $\begin{array}{l}\text { IRAN 3123C; SC } \\
\text { Ahw-Vig-1Rb }\end{array}$ & $\begin{array}{l}\text { Vigna } \\
\text { unguiculata }\end{array}$ & Iran & MG97127 & MG975597 & 7 MH39215 \\
\hline $\begin{array}{l}\text { C. } \\
\text { muehlenbeckiae }\end{array}$ & AR5180 & Sorghum bicolor & Japan & KР400649 & KP419998 & KM196579 \\
\hline $\begin{array}{l}\text { C. } \\
\text { muehlenbeckiae }\end{array}$ & MUS 0031 & Sorghum sp. & USA & KP400647 & KР419996 & KM196578 \\
\hline C. neergaardii & BRIP 12919 & Oryza sativa & Ghana & KJ415550 & KJ415397 & KJ415443 \\
\hline C. neergaardii & DAOM 228085 & desert soil & Chile & KJ909784 & KM083615 & 5 KM196593 \\
\hline C. neoindica & BRIP 17439 & $\begin{array}{l}\text { Trianthema } \\
\text { portulacastrum }\end{array}$ & Australia & AF081449 & AF081406 & - \\
\hline C. nicotiae & BRIP 1 & - & - & KJ415551 & KJ415396 & KJ415442 \\
\hline tiae & CBS & Desert soil & Algeria & 9772 & 33614 & $4-$ \\
\hline C. nisikadoi & CBS 192.29 & - & - & AF081447 & AF081410 & - \\
\hline C. nodosa & СРC 28801 & $\begin{array}{l}\text { Urochloa } \\
\text { reptans }\end{array}$ & Thailand & MF490817 & MF490839 & MF490860 \\
\hline C. nodosa & CPC 28812 & Chloris barbata & Thailand & 0818 & 0840 & MF490861 \\
\hline dulosa & & - & - & 1033 & JN600975 & JN601019 \\
\hline C. oryzae & & Oryza sativa & Vietnam & 400650 & KР645344 & KM196590 \\
\hline ariicola & & - & - & JN601031 & JN600971 & JN601020 \\
\hline C. ovariicola & & - & - & HG778994 & HG779145 & - \\
\hline C. pallescens & & Air & Java & KJ922380 & KM083606 & 6 KM196570 \\
\hline C. papendorfii & BRIP 57608 & Acacia karroo & - & KJ415552 & KJ415395 & KJ415441 \\
\hline C. papendorfii & CBS308.67 & Acacia karroo & $\begin{array}{l}\text { South } \\
\text { Africa }\end{array}$ & KJ909774 & KM083617 & 7 KM196594 \\
\hline C. perotidis & CBS 350.90 & & Cape York & JN192385 & JN601021 & - \\
\hline & & Pisum sativum & Canada & KY905678 & KY905690 & KY905697 \\
\hline C. portulacae & BRIP 14837 & Soil & - & KJ415554 & KJ415392 & KJ415439 \\
\hline C. portulacae & CBS 239.48 & $\begin{array}{l}\text { Portulaca } \\
\text { oleracea }\end{array}$ & USA & KJ909775 & KM083616 & 6 KM230404 \\
\hline C. prasadii & CBS 143.64 & $\begin{array}{l}\text { Jasminum } \\
\text { sambac }\end{array}$ & India & KJ922373 & KM061785 & 5 KM230408 \\
\hline C. prasadii & CBS 144.64 & - & - & HG778997 & HG779149 & \\
\hline C. protuberata & 5876 & Fragaria sp. & - & КТ012665 & KT012626 & KT012587 \\
\hline C. protuberata & CBS 376.65 & $\begin{array}{l}\text { Deschampsia } \\
\text { flexuosa }\end{array}$ & UK & KJ922376 & KM083605 & 5 KM196576 \\
\hline
\end{tabular}


Table 1 Continued.

\begin{tabular}{|c|c|c|c|c|c|}
\hline \multirow[t]{2}{*}{ Species name } & \multirow{2}{*}{$\begin{array}{l}\text { isolate name } \\
\text { strain no.* }\end{array}$} & \multirow{2}{*}{\multicolumn{2}{|c|}{ or Source or host origin }} & \multicolumn{2}{|c|}{ GenBank Accession numbers } \\
\hline & & & & $\begin{array}{ll}\text { ITS } & G P D H \\
\end{array}$ & $E F 1 \alpha$ \\
\hline $\begin{array}{l}\text { C. } \\
\text { pseudobrachyspora }\end{array}$ & CPC 28808 & Eleusine indica & Thailand & MF490819 MF490841 & MF490862 \\
\hline C. pseudolunata & UTHSC 092092 & Nasal sinus & USA & HE861842 HF565459 & - \\
\hline C. pseudorobusta & UTHSC 083458 & Nasal sinus & USA & HE861838 HF565476 & - \\
\hline C. ravenelii & BRIP 13165 & $\begin{array}{l}\text { Sporobolus } \\
\text { fertilis }\end{array}$ & Australia & JN192386 JN600978 & JN601024 \\
\hline C. ravenelii & CBS 127709 & - & - & HG778999 HG779109 & - \\
\hline C. richardiae & BRIP 4371 & $\begin{array}{l}\text { Richardia } \\
\text { brasiliensis }\end{array}$ & Australia & KJ415555 KJ415391 & KJ415438 \\
\hline C. robusta & CBS624 68 & $\begin{array}{l}\text { Dichanthium } \\
\text { annulatum }\end{array}$ & USA & KJ909783 KM083613 & 3 KM19657 \\
\hline C. ryleyi & BRIP 12554 & $\begin{array}{l}\text { Sporobolus } \\
\text { creber }\end{array}$ & - & KJ415556 KJ415390 & KJ415437 \\
\hline C. ryleyi & CBS349.90 & $\begin{array}{l}\text { Sporobolus } \\
\text { creber }\end{array}$ & Yetman & KJ909766 KM083612 & 2 KM19656 \\
\hline C. senegalensis & CBS 149.71 & - & - & HG779001 HG779128 & $3-$ \\
\hline C. senegalensis & ZM02057 & - & - & $7 \mathrm{HC}$ & $3-$ \\
\hline C. soli & CBS 222.96 & soil & $\begin{array}{l}\text { Papua } \\
\text { Guinea }\end{array}$ & KY905679 KY905691 & KY905698 \\
\hline C. sorghina & BRIP 15900 & $\begin{array}{l}\text { Sorghum } \\
\text { bicolor }\end{array}$ & Australia & KJ415558 KJ415388 & KJ415435 \\
\hline C. spicifera & CBS 274.52 & & Spain & JN192387 JN600979 & JN601023 \\
\hline C. subpapendorfii & CBS6 & Desert soil & Egypt & KJ909777 KM061791 & 1 KM196585 \\
\hline C. trifolii & AR5169 & $\begin{array}{l}\text { Sorghum } \\
\text { bicolor }\end{array}$ & South Africa & КР400656 КР645345 & KP735694 \\
\hline C. tripogonis & BRIP 12375 & Unknown & Australia & JN192388J JN600980 & JN601025 \\
\hline C. tropicalis & BRIP 14834 & Coffea arabica & India & KJ415559 KJ415387 & KJ415434 \\
\hline C. tsudae & & Chloris ga & & КC424605 КC747755 & $\mathrm{KC}$ \\
\hline & & Chloris gayana & & KP400651 KM061790 & O KM230409 \\
\hline C. tuberculata & CBS & Zea mays & India & JX256433 JX276445 & JX266599 \\
\hline C. uncinata & CBS 221.52 & Oryza sativa & Vietnam & HG779024 HG779134 & $1-$ \\
\hline C. variabilis & СРC 28813 & $\begin{array}{l}\text { Digitaria } \\
\text { ciliaris }\end{array}$ & Thailand & MF490820 MF490842 & MF49086 \\
\hline C. variabilis & CPC 28815 & Chloris barbata & a Thailand & NR154866 MF490844 & MF490865 \\
\hline C. verruculosa & CBS150 63 & $\begin{array}{l}\text { Punica } \\
\text { granatum }\end{array}$ & India & КР400652 КР645346 & KP735695 \\
\hline & CC 100690 & Oryza sativa & Thailand & & JX266602 \\
\hline & AR5117 & Lolium perene & USA & КР400655 КР645349 & KP735698 \\
\hline Curvularia sp. & MFLUCC 100709 & Oryza sativa & Thailand & JX256442 JX276453 & - \\
\hline Curvularia sp. & MFLUCC 100739 & Oryza sativa & Thailand & JX256443 JX276454 & JX266603 \\
\hline Curvularia sp. & MFLUCC 120177 & Unknown grass & Thailand & КР400654 КР645348 & KP735697 \\
\hline Curvularia sp. & UTHSC 08809 & Human & USA & HE861826 HF565477 & - \\
\hline
\end{tabular}

"BRIP: Queensland Plant Pathology Herbarium, Brisbane, Australia; CBS: Centraalbureau voor Schimmelcultures, Utrecht, The Netherlands; CPC: Culture collection of Pedro Crous, housed at Westerdijk Fungal Biodiversity Institute; DAOM: Plant Research Institute, Department of Agriculture (Mycology), Ottawa, Canada; ICMP: International Collection of Micro-organisms from Plants, Landcare Research, Private Bag 92170, Auckland, New Zealand; IMI: International Mycological Institute, Kew, UK; MAFF: Ministry of Agriculture, Forestry and Fisheries, Tsukuba, Ibaraki, Japan, MFLUCC: Mae Fah Luang University Culture Collection, Chiang Rai, Thailand; UTHSC: Fungus Testing Laboratory, Department of Pathology at the University of Texas Health Science Center, San Antonio, Texas, USA; IRAN: Iranian Fungal Culture Collection, Iranian Research Institute of Plant Protection, Iran; SCUA: the Collection of Fungal Cultures, Department of Plant Protection, Shahid Chamran University of Ahvaz, Iran 
(Schoch et al. 2009). Each polymerase chain reaction (PCR) contained $5 \mu$ of 10x prime Taq Reaction Buffer (GenBio, South Korea), $3 \mathrm{mM} \mathrm{MgCl} 2,0.4 \mathrm{mM}$ dNTPs (mix), $0.4 \mu \mathrm{M}$ of each primer, $0.6 \mu \mathrm{L}$ of Prime Taq DNA Polymerase (5 units/ $\mu \mathrm{l}$, GenetBio), and $5 \mathrm{ng} / \mu \mathrm{L}$ template DNA adjusted with purified water (Mili-Q Water) to a final volume of $50 \mu \mathrm{l}$. Amplification reactions started with an initial denaturation step at $95^{\circ} \mathrm{C}$ for $3 \mathrm{~min}$, followed by 35 cycles of $94{ }^{\circ} \mathrm{C}$ for $30 \mathrm{~s}$, $54{ }^{\circ} \mathrm{C}$ (ITS), $56{ }^{\circ} \mathrm{C}(G P D H)$ or $62{ }^{\circ} \mathrm{C}(E F 1 \alpha)$ for $40 \mathrm{~s}$ and $72{ }^{\circ} \mathrm{C}$ for $1 \mathrm{~min}$. A final elongation step at $72{ }^{\circ} \mathrm{C}$ for $10 \mathrm{~min}$ was set to complete the amplification.

\section{Purification and sequencing}

The PCR products were size-fractionated by gel electrophoresis in 1\% agarose (Hispan Agar) run in 1.0x Tris-acetic acid-EDTA (TAE) buffer stained with commercial safe stain (SinaClon, Iran) and then photographed under UV light. Amplified PCR products of the expected size were excised and purified by GF-1 AmbiClean Kit (Vivantis, Malaysia) according to the manufacturer's instructions. The purified amplicons were read in both directions using original PCR primers by Macrogen Company (Humanizing Genomics, Macrogen, South Korea). BioEdit Sequence Alignment Editor Version 7.0.9.0 (Hall 1999) was used to edit raw ABI chromatograms, after which the forward and reverse sequences were assembled using DNA Baser Sequence Assembler v4 programs (2013, Heracle BioSoft, www.DnaBaser.com). The generated sequences were deposited in GenBank (Table 1).

\section{Phylogenetic analyses}

The nucleotide sequences of ITS, GPDH and EFI $\alpha$ regions were compared to those of reference in GenBank using maximum likelihood (ML) algorithm in MEGA version 6 (Tamura et al. 2013). The combined datasets of ITS-GPDH (two-locus) and ITS-GPDH-EF1 $\alpha$ (three-locus) used for phylogenetic analysis contained the sequence of the isolates under study and reference strains from previously known species of Curvularia, as well as outgroup taxon (Table 1). These reference sequences (Table 1) were mostly from Manamgoda et al. (2015), Madrid et al. (2014), Tan et al. (2014), Marin-Felix et al. (2017a, b). The species of Bipolaris drechsleri was used as outgroup taxon to root phylogenetic trees. Single and multiple sequence alignments were generated with Clustal W in MEGA version 6 (Tamura et al. 2013) using these parameters: pairwise alignment (gap opening 15, gap extension 6.6) and multiple alignment (gap opening 15, gap extension 6.6, transition weight 0.5, delay divergent sequences 30\%). Alignments were checked visually and edited manually where necessary. The phylograms were constructed with best-fitting nucleotide substitution model, as suggested by ML model test using MEGA version 6 (Tamura et al. 2013), Subtree-Pruning-Regrafting (SPR) algorithm and following options: Bootstrap (BP) analyses were done with 1000 replicates, Initial Trees for ML were made by NJ/BioNJ algorithm and Branch Swap Filter was set very strong. Two combined matrix used for phylogenetic analysis were deposited in the Treebase database (http://purl.org/phylo/treebase/phylows/study/TB2: S22797).

\section{Results}

\section{Sequences analysis, BLASTn algorithm and phylogeny}

PCR fragments of approx. $500 \mathrm{bp}$ were successfully amplified for ITS region using primer pair ITS1 and ITS4 and approx. 1100 bp for EF1 $\alpha$ were obtained using the EF1-983 and EF12218R primers pair. Amplicons of approx. $700 \mathrm{bp}$ was successfully produced for GPDH using primers gpd1 and gpd2. The sequences of ITS, GPDH and EF1 $\alpha$ belonging to the isolates under study were submitted to GenBank (Table 1). The isolates of the new described and previously known species of Curvularia shared $74 \%$ sequence identity in the ITS region (468 bp) attributed to 36 SNPs and 82 bp insertion/deletion, 60\% sequence identity in the GPDH region (507 bp) attributed to 86 SNPs and $111 \mathrm{bp}$ insertion/deletion, and 77\% sequence identity in the EF1 $\alpha$ region (677 bp) attributed to 125 SNPs. 
In the BLASTn analysis of the ITS sequence, the closest related species to Curvularia mosaddeghii were C. hominis (HG779011) and C. muehlenbeckiae (HG779002) with 98\% sequence identity. In the analysis of the GPDH, the species with the highest sequence identity to new species were Curvularia hominis (HG779106), Curvularia muehlenbeckiae (HG779108) and Curvularia pisi (KY905690) with 96\%, 95\% and 95\% similarity, respectively. The EF1 $\alpha$ sequences of the new species showed maximum identity to different species, including Curvularia akaiiensis, $C$. australiensis, $C$. buchloes, $C$. chiangmaiensis, $C$. hawaiiensis, C. pisi, C. sorghina, $C$. subpapendorfii, C. tropicalis and $C$. variabilis.

The combined ITS-GPDH and ITS-GPDH-EFI $\alpha$ datasets consisted of 228 and 258 sequences from 114 and 86 taxa including the outgroup taxon, respectively (Table 1). The best model of evolution for phylogenetic analysis was the Tamura-Nei with Invariant Site and Gamma Distribution (TN93 + G + I). ITS-GPDH tree was constructed to show delimitation of new species to some Curvullaria species which their EFl $\alpha$ sequences were not available and excluded in threelocus based tree (not shown, supplemented figure). In both two- and three-locus based trees (Fig. 1), the isolates of Curvularia mosaddeghii formed a monophyletic clade with strong bootstrap 99\% support, distinct from the other previously known species of Curvularia. Phylogenetic analyses based on ITS-GPDH and ITS-GPDH-EF1 $\alpha$ dataset showed that the closest relatives of new species are C. hominis, C. muehlenbeckiae and C. pisi with $73 \%$ and $93 \%$ BS support, respectively (Fig. 1).

\section{Taxonomy}

Curvularia mosaddeghii M. Mehrabi-Koushki \& R. Farokhinejad, sp. nov.,

Fig. 2 MycoBank: MB 825506; Facesoffungi number: FoF04768

Etymology - In reference to Prof. S. Saeed Mosaddegh who significantly contributed to the progress of plant protection science in Khuzestan Province, Iran.

Typification - IRAN, KHUZESTAN PROVINCE: Ahvaz. From jambolan leaf spot (Syzygium cumini), October 2017, K. Heidari and R. Farokhinejad (holotype: IRAN 16938F), extype cultures: IRAN 3131C = SCUA-Ahv-Syz-P).

Morphology on PDA - Hyphae sub-hyaline to brown, branched, septate, thin and smoothwalled, 3-5.25(-7) $\mu \mathrm{m}$ diam. Conidiophores arising singly, septate, generally equal wide in basal parts, tapering in median and upper parts, straight or flexuous, geniculate in apex, frequently unbranched, cells walls thicker than those of the vegetative hyphae, pale brown to brown, 30-161($227) \times(2.6-) 3-5.1 \mu \mathrm{m}, 95 \%$ confidence limits $=77.7-104.8 \times 3.6-4 \mu \mathrm{m},\left(\overline{\mathrm{x}}_{ \pm} \mathrm{SD}=91 \pm 45 \times 3.8 \pm\right.$ $0.6 \mu \mathrm{m}, \mathrm{n}=60)$. Conidiogenous cells mostly integrated, smooth or with verruculose nodes, terminal or intercalary, proliferating sympodially, with circular and thickened scars, brown, cylindrical to swollen. Conidia with bipolar-germination, (1-)4-celled, smooth-walled or slightly verruculose, asymmetrically swollen and curved at the third cell from base, rarely symmetric swelling and straight, pale to dark brown, end cells paler and thin-walled than central cells, 11.1-26.3(-32) $\times$ 7.1-10(-11.1) $\mu \mathrm{m}, 95 \%$ confidence limits $=19.9-22.5 \times 8.6-9.2 \mu \mathrm{m},(\overline{\mathrm{x}} \pm \mathrm{SD}=21.2 \pm 4.3 \times 8.9 \pm$ $1 \mu \mathrm{m}, \mathrm{n}=60)$. Hilum very slightly protruding, darkened, thickened, 1.5-2.3 $\mu \mathrm{m}$ diam. Chlamydospore and sexual morph not observed.

Cultural characters on PDA - Colonies growing 55-60 mm diam after $8 \mathrm{~d}$ incubation at $28 \pm 0.5{ }^{\circ} \mathrm{C}$, circular with filiform margin, dark green to greenish black, aerial mycelium sparse to moderate, floccose with age; reverse greyish green to brownish black.

Habitats - jambolan and cowpea

Distribution - Iran (Ahvaz).

Additional cultures examined - IRAN, KHUZESTAN PROVINCE: Ahvaz. Endophyte in cowpea root (Vigna unguiculata), Sep-2016, S. Janbozorgi and M. Mehrabi-Koushki (IRAN 3123C $=$ SCUA-Ahw-Vig-1Rb). 


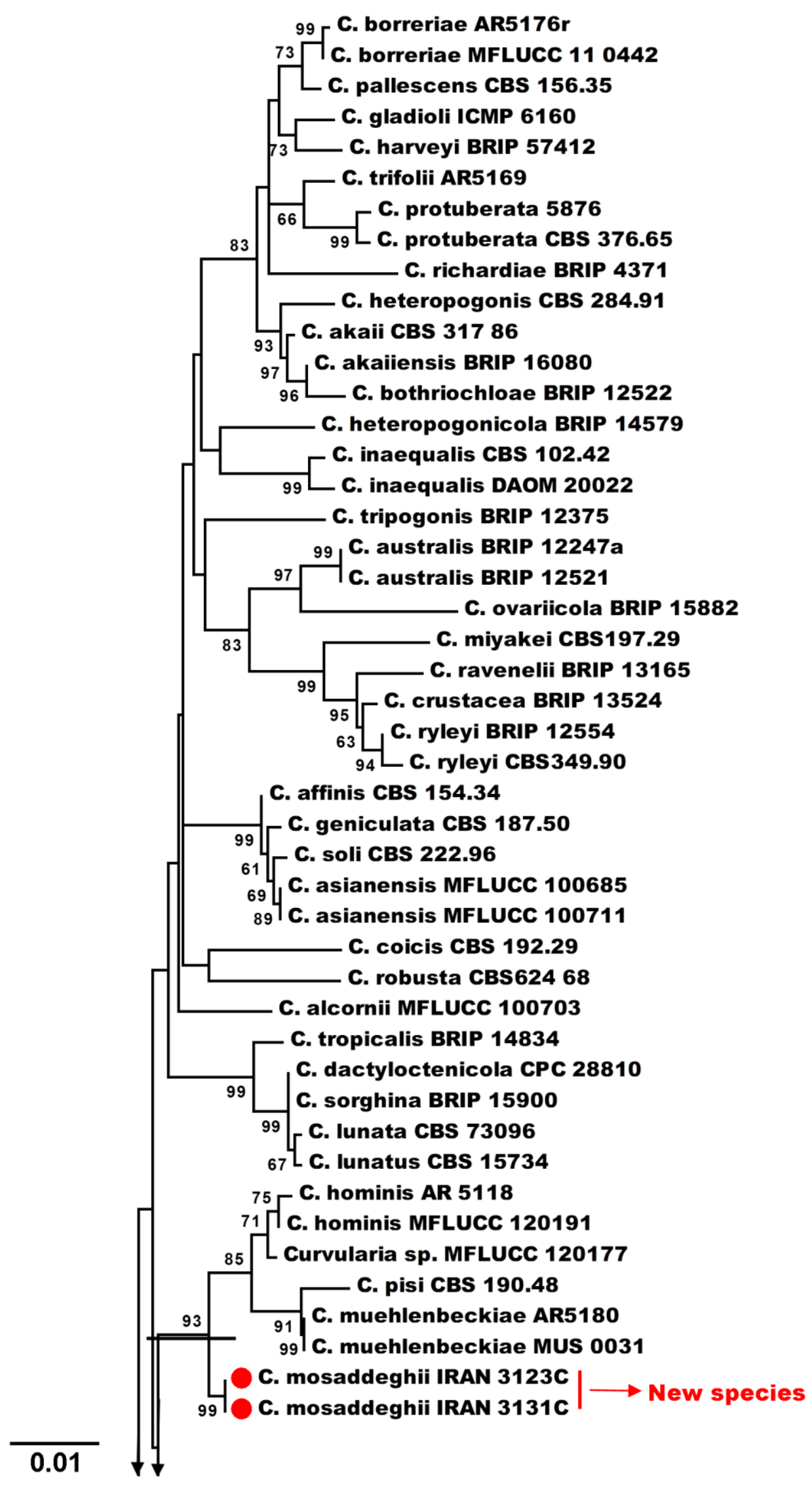

Figure 1 - Phylogenetic tree generated from a ML analysis based on a concatenated alignment of ITS, GPDH and EF1 $\alpha$ sequences of 85 Curvullaria strains representing most previously known species and new taxon. Bootstrap values greater than 50\% (expressed as percentages of 1000 replications) are shown at the nodes. 


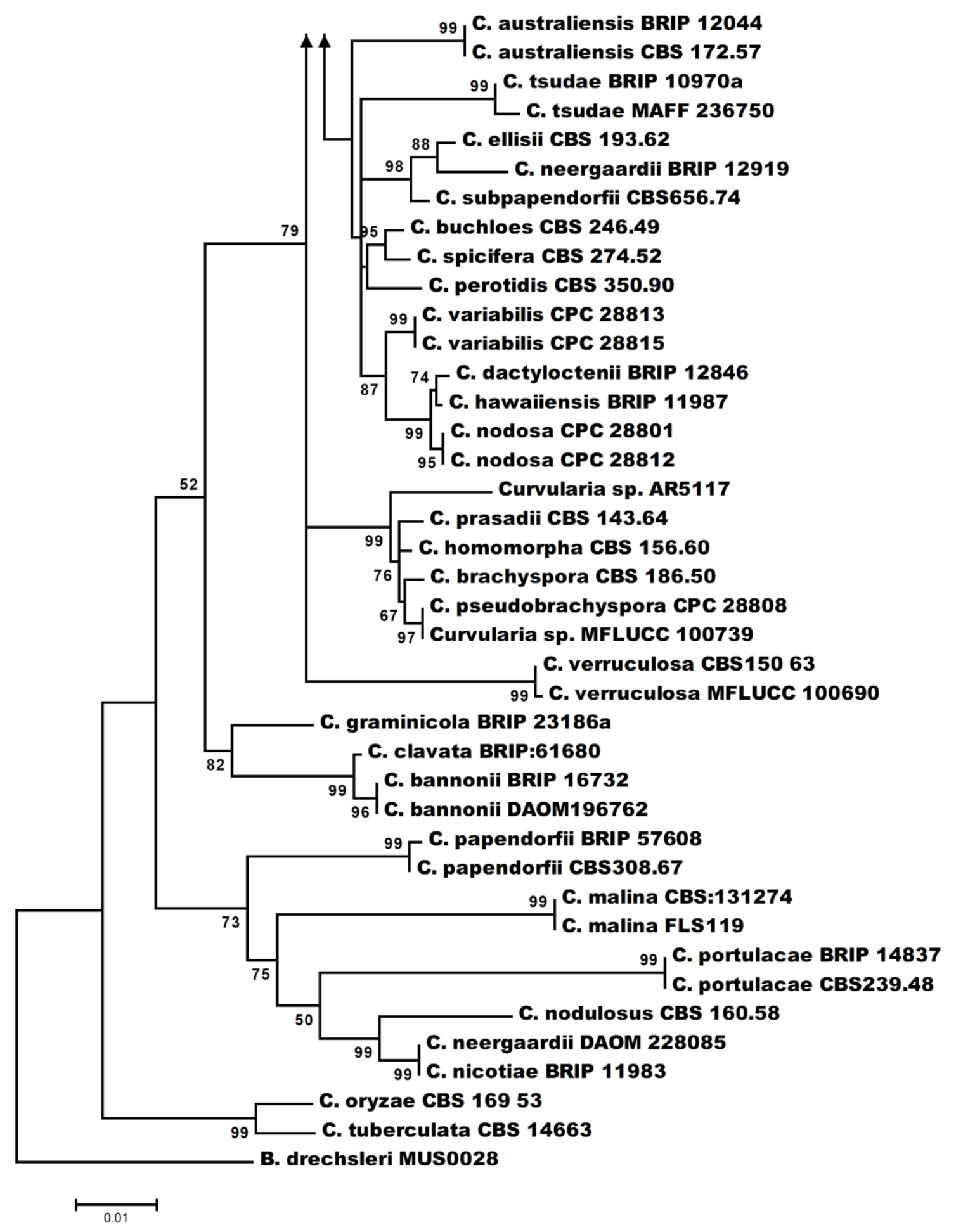

Figure 1 - Continued.

Notes - Curvularia mosaddeghii is slightly different morphologically from closely related species of Curvularia, i.e. C. hominis Da Cunha, Madrid, Gené \& Cano (Madrid et al. 2014), C. muehlenbeckiae Madrid, Da Cunha, Gené, Guarro \& Crous (Madrid et al. 2014) and C. pisi Y. Marin \& Crous (Marin-Felix et al. 2017a). C. mosaddeghii can be easily distinguished from three other species by the production of less wide conidia (7.1-10 $\mu$ m wide vs. $7-14,8.5-12$ and 9-15.5 $\mu \mathrm{m}$ wide in $C$. hominis, $C$. muehlenbeckiae and $C$. pisi, respectively). The conidia in $C$. mosaddeghii, $C$. muehlenbeckiae and $C$. pisi differ from $C$. hominis in being less septate 
(predominantly 3-distoseptate vs. 3-4-distoseptate). In addition, hila in C. mosaddeghii are narrower than the three other species.
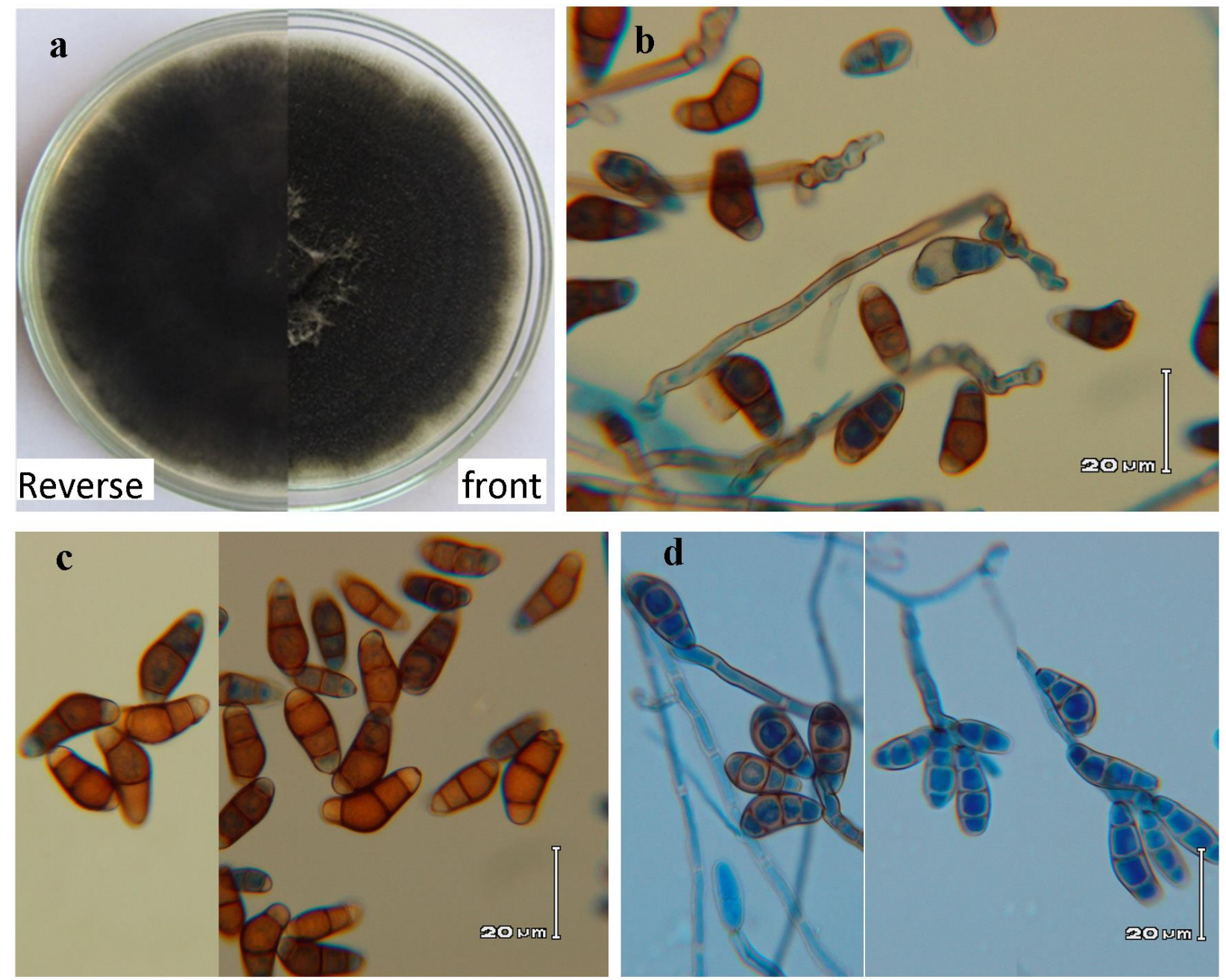

Figure 2 - Curvularia mosaddeghii (IRAN 3131C). a Colony on PDA (front and reverse). b-d Conidiophores and conidia.

\section{Discussion}

In our study, a new species of curvularia is described, which are ascribed to the new species of Curvularia mosaddeghii. Multi-locus phylogenetic analysis and morphology showed that $C$. mosaddeghii isolates are quite distinct from the other previously described Curvularia species, such as $C$. hominis, C. muehlenbeckiae and $C$. pisi. Our phylogenetic analyzes included all the Curvularia taxa used in the studies of Manamgoda et al. (2015), Madrid et al. (2014), Tan et al. (2014), Tomaso-Peterson et al. (2016) and Marin-Felix et al. (2017a, b). In three-locus based phylogenetic tree, the combined dataset (inferred from ITS, GPDH and EF1 $\alpha$ gene sequences) was sufficient to delimit most closely related species in genus Curvularia. Here, the GPDH sequence showed the highest degree of polymorphism among the genomic regions used.

Curvularia mosaddeghii were isolated from jambolan leaf spot and cowpea healthy root, none of its near relatives, including $C$. hominis, $C$. muehlenbeckiae and $C$. pisi, have yet been reported from these hosts. Curvularia hominis and $C$. muehlenbeckiae have been isolated from human tissues and Muehlenbeckia sp. leaf in USA, respectively (Madrid et al. 2014). C. pisi was reported from the seeds of Pisum sativum in Canada (Marin-Felix et al. 2017a). Curvularia mosaddeghii is known to be as endophyte within Vigna unguiculata, which is also host for three other species of Curvularia, C. eragrostidis (Henn.) J.A. Mey., C. lunata (Wakker) Boedijn and C. verruculosa Tandon \& Bilgrami (Ahmad et al., 1993, Manamgoda et a., 2011, Mogle and Maske 
2012, Abdulwehab et al. 2015). However, these species are morphologically and phylogenetically different to C. mosaddeghii. Morphologically, these species differ from C. mosaddeghii in having wider conidia.

\section{Acknowledgment}

This work was financially supported by grants from Research Council of Shahid Chamran University of Ahvaz.

\section{References}

Abdulwehab SA, El-Nagerabi SAF, Elshafie AE. 2015 - Leguminicolous fungi associated with some seeds of Sudanese legumes. Biodiversitas 16, 269-280.

Ahmad I, Iftikhar S, Bhutta AR. 1993 - Seed-borne microorganisms in Pakistan, Check List 1991. Pakistan Agricultural Research council. Islamabad. 32 pp.

Ahmadpour SA, Mehrabi-Koushki M, Farokhinejad R. 2017 - Neodidymelliopsis farokhinejadii, a new fungal species from dead branches of trees in Iran. Sydowia 69,171-182

Ariyawansa HA, Thambugala K, Manamgoda DS, Jayawardena R et al. 2015 - Towards a natural classification and backbone tree for Pleosporaceae. Fungal Diversity 71, 85-139.

Beneke ES, Rogers AL. 1996 - Medical mycology and human mycoses. Star Publishing Company, Belmont, $239 \mathrm{pp}$.

Berbee M, Pirseyedi M, Hubbard S. 1999 - Cochliobolus phylogenetics and the origin of known, highly virulent pathogens, inferred from ITS and glyceraldehyde-3-phosphate dehydrogenase gene sequences. Mycologia 91, 964-977.

Boedijn KB. 1933 - Ueber einige phragmosporen Dematiazeen. Bulletin du Jardin botanique de Buitenzorg 13, 120-134.

da Cunha KC, Sutton DA, Fothergill AW, Gene J, Cano J, Madrid H, de Hoog S, Crous PW, Guarroa J. 2013 - In vitro antifungal susceptibility and molecular identity of 99 clinical isolates of the opportunistic fungal genus Curvularia. Diagnostic Microbiology and Infectious Disease 76, 168-174.

Gautam AK, Kant M, Thakur Y. 2013 - Isolation of endophytic fungi from Cannabis sativa and study their antifungal potential. Archive of Phytopathology and Plant Protection 46, 627-635.

Hall TA. 1999 - BioEdit: a user-friendly biological sequence alignment editor and analysis program for windows 95/98/NT. Nucleic Acids Symposium Series 41, 95-98.

Hyde KD, Nilsson RH, Alias SA, Ariyawansa HA et al. 2014 - One stop shop: backbones trees for important phytopathogenic genera: I. Fungal Diversity 67, 21-125.

Jena SK, Tayung K. 2013 - Endophytic fungal communities associated with two ethno-medicinal plants of Similipal Biosphere Reserve, India and their antimicrobial prospective. Journal of Applied Pharmaceutical Science 3, 7.

Kornerup A,Wanscher JH. 1967 - Methuen handbook of colour. Methuen \& Co. Ltd, London

Madrid H, da Cunha KC, Gene J, Dijksterhuis J, Cano J, Sutton DA, Guarro J, Crous PW. 2014 Novel Curvularia species from clinical specimens. Persoonia 33, 48-60.

Manamgoda DS, Cai L, Bahkali AH, Chukeatirote E, Hyde KD. 2011 - Cochliobolus: an overview and current status of species. Fungal Diversity 51, 3-42.

Manamgoda DS, Cai L, McKenzie EH, Crous PW, Madrid H, Chukeatirote E, Shivas RG, Tan YP, Hyde KD. 2012a - A phylogenetic and taxonomic re-evaluation of the BipolarisCochliobolus-Curvularia complex. Fungal Diversity 56, 131-44.

Manamgoda DS, Cai L, McKenzie EHC, Chukeatirote E, Hyde KD. 2012b - Two new Curvularia species from northern Thailand. Sydowia 64, 255-266.

Manamgoda DS, Rossman AY, Castlebury LA, Chukeatirote E, Hyde KD. 2015 - taxonomic and phylogenetic re-appraisal of the genus Curvularia (Pleosporaceae): human and plant pathogens. Phytotaxa 212, 175-198. 
Manamgoda DS, Rossman AY, Castlebury LA, Crous PW, Madrid H, Chukeatirote E, Hyde KD. 2014 - The genus Bipolaris. Studies in Mycology 79, 221-288.

Marin-Felix Y, Groenewald JZ, Cai L, Chen Q, Marincowitz S et al. 2017a - Genera of phytopathogenic fungi: GOPHY 1. Studies in Mycology 86, 99-216.

Marin-Felix Y, Senwanna C, Cheewangkoon R, Crous PW. 2017b - New species and records of Bipolaris and Curvularia from Thailand. Mycosphere 8, 1556-1574.

Mogle UP, Maske SR. 2012 - Efficacy of bioagents and fungicides on seed mycoflora, germination and vigour index of cowpea. Science Research Reporter 2, 321-326.

Rangaswamy BE, Francis F, Prakash KK, Manjunath NS. 2013 - Variability in airborne bacterial and fungal population in the tertiary health care centre. Aerobiologia 29, 473-479.

Raeder U, Broda P. 1985 - Rapid preparation of DNA from filamentous fungi. Letters in Applied Microbiology 1, 17-20.

Riddle OC, Briggs FN. 1950 - Inheritance of resistance to scald in barley. Hilgardia 20, 19-27.

Schoch C, Crous PW, Groenewald J, Boehm E, Burgess TI, De Gruyter J, De Hoog G, Dixon L, Grube M, Gueidan C. 2009 - A class-wide phylogenetic assessment of Dothideomycetes. Studies in Mycology 64, 1-15.

Scott EM, Carter RT. 2014 - Canine keratomycosis in 11 dogs: A case series (2000-2011). Journal of the American Animal Hospital Association 50, 112-118.

Sivanesan A. 1987 - Graminicolous species of Bipolaris, Curvularia, Drechslera, Exserohilum and their teleomorphs. Mycological Papers 158, 1-261.

Tadych M, Bergen M, Johnson JC, Polashock J, Vorsa N. 2012 - Endophytic and pathogenic fungi of developing cranberry ovaries from flower to mature fruit: diversity and succession. Fungal Diversity 54, 101-116.

Tamura K, Stecher G, Peterson D, Filipski A, Kumar S. 2013 - MEGA6: molecular evolutionary genetics analysis version 6.0. Molecular Biology and Evolution 30, 2725-2729.

Tan YP, Madrid H, Crous PW, Shivas RG. 2014 - Johnalcornia gen. et. comb. nov., and nine new combinations in Curvularia based on molecular phylogenetic analysis. Australasian Plant Pathology 43, 589-603.

Tomaso-Peterson M, Jo YK, Vines PL, Hoffmann FG. 2016 - Curvularia malina sp. nov. incites a new disease of warm-season turfgrasses in the southeastern United States. Mycologia 108, 915-924.

Verma P, Singh S, Singh R. 2013 - Seven species of Curvularia isolated from three lakes of Bhopal. Advances in Life Science and Technology 8, 13-15.

White TJ, Bruns T, Lee S, Taylor J. 1990 - Amplification and direct sequencing of fungal ribosomal RNA genes for phylogenetics. In: Innis MA,Gelfand DH, Sninsky JJ, White TJ (eds) PCR protocols: a guide to methods and applications. Academic Press, New York, pp 315-322.

Wijayawardene NN, Hyde KD, Rajeshkumar KC, Hawksworth DL et al. 2017 - Notes for genera: Ascomycota. Fungal Diversity 86, 1-594.

Wijayawardene NN, Hyde KD, Lumbsch T, Liu JK et al. 2018 - Outline of Ascomycota - 2017. Fungal Diversity 88, 167-263. 